\title{
VII. Überblick
}

Eine wichtige Dimension der in dieser Studie thematisierten Auseinandersetzung waren die "nationalen Verhältnisse“ in der Wirtschaft: die nationale Zusammensetzung der Belegschaften, insbesondere in den industriellen Großbetrieben der Republik. In nationalpolitischer Hinsicht war hier vor allem der Umstand von Bedeutung, daß Deutsche zwischen 1918 und 1938 in den oberen Hierarchierängen tschechoslowakischer Betriebe, nicht zuletzt in den bedeutenden Unternehmen der Chemie, der Elektrotechnik, der Eisen- und der Metallindustrie sowie des Kohlebergbaus großes Gewicht als Techniker und Ingenieure, Verwaltungsangestellte, leitende Manager bzw. Direktoren besaßen. Einige dieser Deutschen waren Einheimische, also tschechoslowakische Staatsbürger, viele waren Deutsch-Österreicher; es fand sich aber auch eine größere Anzahl von Reichsdeutschen, d. h. von Bürgern desjenigen Landes, von dem viele Tschechen eine Unterjochung ihrer Republik am ehesten befürchteten. Tendenziell verschwammen in der Wahrnehmung die Unterschiede zwischen diesen Segmenten, manchmal wurden sie aus politischen Absichten auch bewußt verwischt.

Daß in- oder ausländische Deutsche die Kommandohöhen ausgerechnet der modernsten und produktivsten Betriebe der Republik besetzten, war im wesentlichen eine Erbschaft aus der Zeit der Monarchie. Die anvisierte Gruppe umfaßte vermutlich nicht mehr als etwa 30000 Personen; präzisere statistische Angaben existieren nicht. Nicht korrekt wäre es, sie als „Elitekorps“ zu bezeichnen: Die Vernetzung dürfte eher locker gewesen sein, für die etwa vom Nationalrat unterstellte „bewußte Kolonienbildung in konspirativer Absicht" fehlen Hinweise völlig. Für viele nationalbewußte Tschechen war die Existenz dieses Personenkreises trotzdem schwer erträglich: Fehlte ihrer Meinung nach damit doch ein gutes Teil des Fundaments der mühsam errungenen politischen Selbständigkeit der Tschechoslowakei, nämlich die de facto-Verfügungsmacht des Staatsvolkes über die Wirtschaft. Die Bedrohung nahm sich umso größer aus, als sich diese Konzentration deutscher Kräfte auf den höheren Hierarchieebenen nicht nur der als deutsch firmierenden Betriebe fand: die Lage war in tschechischen oder national nicht zurechenbaren Unternehmen kaum anders. Besonders delikat war die starke Position der Deutschen in verteidigungswichtigen Betrieben; der Aspekt der nationalen Sicherheit - die Beschäftigung national und politisch eventuell „unzuverlässiger" Personen in den Schlüsselstellungen verteidigungswichtiger Betriebe - hatte bereits in den zwanziger Jahren vor allem in den militärischen Kreisen und dem eng mit diesen verflochtenen Nationalrat eine Rolle gespielt. Er trat mit dem wachsenden Schatten des nationalsozialistischen Regimes und der zunehmenden Hinwendung der Deutschen im Lande zu diesem vor allem in der zweiten Hälfte der dreißiger Jahre noch weiter in den Vordergrund. Zu dieser "nationalitätenpolitischen Frage“ im engeren Sinne kamen, vor allem in den krisenhaften Anfangsjahren der Ersten Republik und wieder im Zeichen der Weltwirtschaftskrise, heftige Verteilungskämpfe zwischen den deutschen Fachkräften und dem tschechischen akademischen Nachwuchs um die knappen hochqualifizierten Arbeitsplätze. Nicht zuletzt war die Arbeitsmarktpolitik gegenüber den Deutschen und Österreichern im eigenen Lande aber auch als Reaktion auf die oft unfreundliche Arbeitsmarktpolitik des Reiches - bzw. Österreichs - gegenüber der nicht 
unbeträchtlichen Gruppe tschechoslowakischer Staatsbürger in diesen beiden Ländern zu verstehen.

Die tschechoslowakische Arbeitsmarktpolitik neigte unter dem Einfluß der öffentlichen Meinung, der Volkstumsorganisationen, der Lobby der tschechischen Privatangestellten und Ingenieure, nicht zuletzt auch unter dem Druck des Militärs zu - je nach den wirtschaftlichen und politischen Rahmenbedingungen - mehr oder weniger energischen Restriktionen der Ausländerbeschäftigung, speziell zur Zurückdrängung und Ersetzung der in leitenden Positionen der Wirtschaft bereits beschäftigten Deutschen. Sie unterstützte auch Maßnahmen zur Abdrosselung eines weiteren Zustroms. „Volkstumspolitische", gesamtwirtschaftliche, militärische und Verbandsinteressen waren hierbei in schwerlich zu entflechtender Weise verquickt. Die Frage der "nationalen Verhältnisse" wurde zum ersten Mal nach 1918 virulent im Zusammenhang mit der Nostrifizierung, der Politik also, mit der die Prager Regierungen, um die wirtschaftspolitische Souveränität zu erringen, auf dem Territorium der Republik operierende Unternehmen zur Verlegung ihrer Unternehmenssitze - meist von der "alten Hauptstadt Wien “ - in die ČSR zu veranlassen suchten. Dies ging Hand in Hand mit Bestrebungen zur Ausweitung des tschechischen Kapitaleinflusses; mit diesem Hebel sollte dann auch eine Umschichtung der nationalen Verhältnisse bewirkt werden. Der „Ausweisungskrieg“ mit Deutschland von 1923 wurde allerdings, ungeachtet einer den Deutschen wenig wohlgesonnenen Stimmung in der Tschechoslowakei, von der Gegenseite vom Zaun gebrochen. Hier wie im Jahr 1928, als nun die Tschechoslowakei ihrerseits gewisse, offensichtlich in erster Linie auf die „Problemgruppe“ der Reichsdeutschen und Deutschösterreicher gezielte rechtliche und praktische Restriktionen der Ausländerbeschäftigung einzuführen suchte, wurde eine Eskalation schließlich auf dem Wege bilateraler tschechoslowakischdeutscher Verhandlungen vermieden. Die dreißiger Jahre verschärften den Konflikt. Der restriktive Kurs der ČSR ist nun zu einem wesentlichen Teil als Retorsionsmaßnahme gegen die rigide, ja brutale Entfernung tschechoslowakischer Staatsangehöriger - oft solche deutscher Volkszugehörigkeit - aus der reichsdeutschen Wirtschaft nach 1933 zu erklären. Das höchste Stadium des Konflikts war mit dem Staatsverteidigungsgesetz von 1936 erreicht, das die Eingriffsrechte des Militärs in die Wirtschaft ausweitete und in diesem Zusammenhang auch die Zugriffsmöglichkeiten der Behörden auf die Personalpolitik der Unternehmen in bis dahin ungeahnter Weise verstärkte.

Die Politik, die auf die Zurückdrängung der Deutschen aus den leitenden Positionen in der Privatwirtschaft zielte, zeitigte - in den dreißiger mehr als in den zwanziger Jahren - signifikante, von manchen Deutschen mit Erbitterung auf dem Konto der „Tschechisierung " verbuchte Resultate. Die Entwicklung in diesem strategisch zentralen Segment des Arbeitsmarkts verschärfte also zweifellos die wirtschaftlichen und politischen Spannungen zwischen Tschechen und Deutschen. Daß von einer durchgreifenden "ethnischen Säuberung“ der Betriebe bis zum Schluß, also sogar unter der Ägide des Staatsverteidigungsgesetz trotzdem nicht die Rede sein konnte, war in erster Linie auf folgende Gründe zurückzuführen: Zum einen lag der Arbeitsmarktpolitik kein einheitlich und umfassend konzipierter, von den wesentlichen gesellschaftlichen und politischen Interessengruppen durchgängig getragener und konsequent durchgeführter "Master plan“ zugrunde. Besonders beliebt war die nationalpolitische Karte bezeichnenderweise in Wahlkampfzeiten. Zum anderen waren die potentiell Betroffenen in vielen Fällen aus betriebswirtschaftlichen oder technischen Gründen schlichtweg überhaupt nicht oder 
nur mit größeren Komplikationen zu ersetzen. Hier war in vielen Fällen offensichtlich die "List der Vernunft“ am Werk: War die Einräumung von Kapitalbeteiligungen an tschechoslowakische Banken ursprünglich dazu gedacht, die nationaltschechische Kontrolle über deutsche Unternehmen zu verstärken und das deutsche Element dort zurückzudrängen, so wirkte sie faktisch dahingehend, daß das Interesse auch der tschechischen Kapitaleigner an einem reibungslosen Ablauf der Produktion erhöht, dasjenige an einer Änderung der "nationalen Verhältnisse“ aber reduziert wurde. Ganz allgemein gilt, daß die deutsche wie die tschechische Industrie des Landes sich sehr skeptisch zeigte gegenüber allen die Existenz ihrer Manager, Direktoren und Experten gleich welcher Nationalität bedrohenden Maßnahmen. Hinzu kam, daß der Rechtsstaat der Ersten Republik willkürliche Eingriffe der Behörden in die Sphäre der Privatwirtschaft zwar nicht ganz, aber doch in sehr weitgehendem Maße verhinderte. Gegen eine allzu restriktive Regelung der Ausländerbeschäftigung allgemein und gegen eine rigorose Durchführung des Staatsverteidigungsgesetzes im besonderen sprach auch das Risiko internationaler Komplikationen. Vor allem die in vergleichsweise großer Zahl im Reich bzw. in Österreich beschäftigten tschechoslowakischen Staatsbürger hatten in diesem Zusammenhang geradezu die Funktion von "Geiseln“. Bezeichnenderweise waren die legislativen Zäsuren der Arbeitsmarktpolitik - 1923, 1928/29 und 1936 - durchwegs unmittelbar oder mittelbar außenpolitisch motiviert; die einschlägigen Regelungen beruhten zu einem guten Teil nicht auf innerstaatlichem Recht, sondern auf bilateralen Abmachungen. 